\title{
Synopsis to: Facile Synthesis and Detailed Characterisation of a new Ferrocenyl Uracil Peptide Nucleic Acid Monomer
}

By

Gilles Gasser, Matthew J. Belousoff, Alan M. Bond and Leone Spiccia

A new ferrocenyl uracil Peptide Nucleic Acid (PNA) hybrid has been developed which binds to 9-ethyladenine (EA), a model for the complementary DNA nucleobase adenine, with sufficient affinity and selectivity to be exploited in electrochemical biosensor applications.



